\title{
Minitrack on Smart City Digital Twins
}

\author{
John E. Taylor \\ Georgia Institute of Technology \\ jet@gatech.edu
}

\author{
Gisele Bennett \\ Florida Institute of Technology \\ gbennett@fit.edu
}

\author{
Neda Mohammadi \\ Georgia Institute of Technology \\ nedam@gatech.edu
}

Digital Twins are an endeavor to create intelligent adaptive machines by generating a parallel virtual version of the system along with the connectivity, analytical, and visualization capabilities enabled by Internet of Things (IoT) and emerging virtualization technologies such as Virtual Reality (VR), Augmented Reality (AR), and Mixed Reality (MR). In this Minitrack we adopt this framework as the foundation for the development, predictive analytics, and adaptive capabilities of Smart City Digital Twins [1].

Emerging interdependencies between humans, infrastructures, and technologies as cities implement socio-technical advancements to transform into smarter cities may result in increasing uncertainties, unreliable predictions, and poor management decisions. Decision makers in cities require an enhanced ability to model, understand, and anticipate the operational dynamics across human, infrastructure, and technology systems collectively as an integrated entity. However, we lack insights into system behavior differences at and across scales in relation to city infrastructure systems. Lack of scalable data integration approaches that take into account the space-time fluctuations across scales add to the complexity of understanding and managing the complex decision making processes of cities. Although sensors provide data, sensor data only gives us an abstract view of operations, at best. Nuances of human experience are concealed beyond the sensors' monitoring reach. Understanding these complexities requires a paradigm shift in how city dynamics are understood, influenced, and ultimately managed. Smart City Digital Twins enable simulation of what-if scenarios through integrated cyber-physical city infrastructures that are embedded with information about the dynamics of infrastructure processes, environmental qualities, and human activities, as well as adaptation to changing conditions. This can help analysts and decision makers perceive interdependencies, anticipate emergent behavior, understand how cities equipped with smart technologies will likely perform under various economic, environmental, and social conditions, and identify the drivers of possible disruptions. Such understanding is critical in assessing whether or not smart growth strategies are effective, minimizing the gap between smart utopia and smart reality. This is a highly complex task that requires new technological and methodological advancements and collaborative participation from various disciplines.

This Minitrack establishes the theoretical and scholarly foundation of a Smart City Digital Twin paradigm that enables increased visibility into cities' human-infrastructure-technology interactions through learning, analytics and exchange of spatiotemporal data in a city. Six exemplar papers addressing both theoretical and practical aspects of Smart City Digital Twins with particular emphasis (but not limited) to the following topics are featured in this edition of the minitrack at HICSS-52:

- Theory, Scale, and System Architecture for Smart City Digital Twins.

- Data, Sensing, IoT, and Analytics for Smart City Digital Twins.

- Human-Infrastructure Interdependencies, Connectivity, and Citizen Engagement.

- Information Management and Decision Support for Smart City Digital Twins.

- Digital Twin virtualization (Virtual Reality / Augmented Reality / Mixed Reality) of Smart Cities.

- Implications for Operational Readiness, ContextAware Simulation, and Crisis Management.

\section{References}

[1] Mohammadi N, Taylor JE. Smart City Digital Twins. IEEE Symp. Ser. Comput. Intell., 2017.

doi:10.1109/SSCI.2017.8285439. 